\title{
Lobna Mestaoui, Tradition orale et esthétique romanesque: aux sources de l'imaginaire de Kourouma
}

\section{Alexandre Calvanese}

\section{(2) OpenEdition}

1 Journals

\section{Édition électronique}

URL : http://journals.openedition.org/studifrancesi/1651

DOI : 10.4000/studifrancesi. 1651

ISSN : 2421-5856

Éditeur

Rosenberg \& Sellier

\section{Édition imprimée}

Date de publication : 1 novembre 2014

Pagination : 644-645

ISSN : 0039-2944

\section{Référence électronique}

Alexandre Calvanese, «Lobna Mestaoui, Tradition orale et esthétique romanesque: aux sources de l'imaginaire de Kourouma », Studi Francesi [En ligne], 174 (LVIII | III) | 2014, mis en ligne le 01 novembre 2014, consulté le 18 septembre 2020. URL : http://journals.openedition.org/studifrancesi/1651 ; DOI : https://doi.org/10.4000/studifrancesi.1651

Ce document a été généré automatiquement le 18 septembre 2020.

\section{(c)}

Studi Francesi è distribuita con Licenza Creative Commons Attribuzione - Non commerciale - Non opere derivate 4.0 Internazionale. 


\title{
Lobna Mestaoui, Tradition orale et esthétique romanesque: aux sources de l'imaginaire de Kourouma
}

\author{
Alexandre Calvanese
}

\section{RÉFÉRENCE}

LOBNA MESTAOUI, Tradition orale et esthétique romanesque: aux sources de l'imaginaire de Kourouma, Paris, L’Harmattan, 2012 (coll. «Histoire, Textes, Sociétés»), pp. 334.

1 Le rapport entre tradition orale et écriture romanesque a été l'objet de plusieurs études dans le cadre de la littérature critique sur Ahmadou Kourouma, mais cet ouvrage de Lobna MESTAOUI a le mérite de reconsidérer la matière de manière systématique et approfondie, sans se borner à une simple constatation de l'existence de ce lien ou à une recension des références orales dans le texte écrit, mais en focalisant l'attention - pour reprendre les mots de la préface signée par Jean DERIVE - sur «l'écart entre leur fonction et leur forme dans la culture orale originelle et la forme et la fonction qu'elles prennent dans les trois romans étudiés» (p. 14). Le corpus de base - formé par les trois premiers romans de Kourouma: Les Soleils des indépendances, Monné, outrages et défis, En attendant le vote des bêtes sauvages - a été sélectionné pour des raisons de cohérence interne et de continuité thématique, à savoir le fait que «les trois héros de ces œuvres, Fama, Djigui et Koyaga, incarnent l'homme politique pris dans différentes postures» (p. 20), et que cette figure n'est pas sans en rappeler une autre, celle du héros épique, dont la réactualisation se traduit en une critique des réalités modernes.

2 L'étude se compose de trois parties. La première, intitulée «Personnages romanesques et archétypes oraux» (pp. 29-118), propose une analyse des personnages kouroumiens en repérant les filiations avec certains modèles omniprésents dans les productions orales tels que le héros chasseur, le héros traditionnel et le héros fondateur. Selon MESTAOUI, En attendant le vote des bêtes sauvages est le roman de Kourouma dans lequel 
l'emprunt au modèle épique universel se fait le plus patent, aussi bien que l'ancrage à la tradition des chants cynégétiques de la culture malinké. Kourouma exploite dans son roman un certain nombre de motifs récurrents du récit épique: l'ascendance hors du commun du héros (Tchao, le père de Koyaga, est le plus grand champion de luttes initiatiques que son peuple ait connu, et Nadjouma, sa mère, est la championne féminine); la séquence du mariage-rapt qui prélude à la grossesse extraordinaire de Nadjouma et à la naissance d'un bébé de taille et de force exceptionnelles; les prouesses de l'enfance qui annoncent la soif inextinguible de pouvoir du futur dictateur; l'expérience de l'exil qui prépare à une nouvelle naissance; enfin, l'épreuve des «monstres», qui évoque les exploits de Thésée ou d'Héraclès, l'épopée de Gilgamesh et, encore - confirmant ainsi l'existence d'archétypes partagés par des foyers culturels éloignés les uns des autres - l'univers de la chasse africaine. Kourouma travaille ce répertoire en proposant une réécriture qui combine l'espace traditionnel de la brousse avec l'espace moderne de la ville, l'affrontement avec le fauve monstrueux (dans la brousse) n'étant qu'une épreuve de préparation à l'affrontement que Koyaga devra soutenir face à ses rivaux politiques (dans la ville). Dans Les Soleils et dans Monné, bien que l'imprégnation épique soit moins visible, la caractérisation des personnages ne lui est pas moins redevable. Fama et Djigui appartiennent tous les deux à une sphère royale, la prédestination joue un rôle essentiel dans leur vie, et tous les deux subissent la spoliation et la destitution par un ennemi. Mais, à la différence du héros épique, pour eux «la reconquête est impossible car aussi bien le personnage que sa communauté évoluent dans un univers divisé, livré à l'arbitraire, héritage du colonialisme et du néocolonialisme» (p. 56). En conséquence de ce déchirement, le personnage romanesque se présente comme fortement individualisé et, au lieu de représenter toute une communauté, il entretient des relations conflictuelles avec elle. La même démarche analytique - définition d'un archétype dans les récits oraux, repérage des éléments romanesques issus de ce modèle et des éléments novateurs par rapport à ce dernier est employée pour montrer la présence du mythe de fondation dans les trois romans de Kourouma, en particulier à travers la figure du marabout. Complètent ce premier chapitre: une réflexion sur le concept de double (ombre de l'individu après sa mort, ou alter ego du héros pendant sa vie), et un portrait de la femme kouroumienne au regard de la tradition, où les trois protagonistes féminines de l'œuvre de Kourouma - Salimata, Moussokoro e Nadjouma - sont respectivement interprétées comme hypostase de la divinité primitive, comme manifestation de la mère dévorante et comme figure de la mère initiatrice.

Dans la deuxième partie, intitulée "Temps et espace» (pp. 119-208), Mestaoui se propose «d'approcher le temps et l'espace comme des chronotopes culturels attachés à une aire géographique donnée, l'Afrique subsaharienne des ethnies mandes, malinké plus précisément, à partir de la représentation qu'en donnent les romans de Kourouma» (p. 122). Dans Les Soleils la convocation du temps sacré est fonctionnelle à la constitution d'un «tableau de la dépravation qui sévit dans l'univers postcolonial» (p. 125) et en particulier dans la ville, où l'individualisme est étroitement associé à l'émancipation des valeurs ancestrales. Une dégradation qui se fait encore plus grave dans En attendant le vote des bêtes sauvages, où les fêtes religieuses se trouvent inscrites dans le sillage de la propagande politique, et où les pratiques ancestrales sont réactualisées pour "pérenniser le chaos moderne» (p. 154) et préserver l'hégémonie du pouvoir de Koyaga. De manière analogue, si le recours au paradigme du temps cyclique et de la répétition d'une part peut être synonyme de réversibilité et de plénitude 
sacrale (en opposition à la conception occidentale du temps), de l'autre il serait figure de l'impuissance des personnages à dépasser leur situation, de l'impossibilité de l'avenir, du non-sens de l'ère moderne. C'est dans le cadre de ces constatations que Mestaoui affirme que la «nostalgie de l'archaïque» se manifeste partout dans l'œuvre de Kourouma, aussi bien dans sa capacité de faire revivre une conception archaïque que dans l'emploi de son symbolisme au service d'un discours critique qui vise les régimes autoritaires. Une lecture qui s'oppose ainsi à celle qui voit en Kourouma un écrivain impartial dans sa satire amère de la société africaine, que ce soit celle des «soleils des indépendances» ou celle de l'époque d'avant la colonisation. $\mathrm{Si}$, par rapport à la catégorie du temps, Mestaoui montre que l'opposition entre passé et présent est assez nette (Kourouma songerait au passé comme à l'âge d'or, alors que le présent est totalement négatif), son analyse de la conception de l'espace africain dans l'œuvre de Kourouma fait ressortir une ambivalence de fond. Par exemple, dans l'opposition structurelle entre brousse et village, la première s'impose tantôt comme l'ailleurs lointain et redoutable, tantôt comme refuge pour l'individu pourchassé ou persécuté, tandis que le deuxième représente la bipartition entre sacré maîtrisé et sacré non maitrisé, ou si l'on veut entre le centre qui abrite tout ce qui incarne le sacré dans ses acceptions positives et la périphérie qui abrite des forces sinistres et centrifuges. Dans les parties consacrées à l'analyse de la représentation des éléments naturels, MESTAOUI met l'accent sur la composante négative de l'ambivalence: «la boue s'impose comme l'archétype de l'hybridité malsaine» (p. 178), «l'eau prend une part déterminante dans l'évocation de la déliquescence des réalités africaines» (p. 179), la force du vent représente «la violence des réalités africaines» (p. 183), «le feu de brousse s'en tient souvent à métaphoriser la destruction, comme si toute renaissance semblait hors de portée» (p. 187). De manière analogue, MESTAOUI relève que chaque fois que Kourouma s'intéresse au règne animal «c'est pour insister sur deux traits qui règlent le régime de ses apparitions: l'agitation vaine et hostile et l'agressivité sadique» (p. 188). La section finale de cette deuxième partie propose d'identifier quelques nouveaux chronotopes corrélés à l'avènement de la colonisation: la route, le chemin de fer, la prison et la radio. Parfois Mestaoui semble plus intéressée à saisir la valeur documentaire du texte, à trouver dans la fiction une confirmation aux données extratextuelles d'ordre ethnologique ou anthropologique, comme si, en quelque sorte, il était nécessaire de justifier l'intérêt pour le texte littéraire en le rattachant à autre chose. C'est ainsi qu'en parlant d'un lieu objet de sacralisation dans le Mandingue, elle affirme: «Que Kourouma ne s'attarde pas sur cet espace n'amoindrit en rien son importance dans la vie des villageois» (p.170), sans expliquer en quoi cela devrait augmenter son intérêt spécifiquement littéraire.

4 La troisième partie, «Modes, formes et structures d'une oralisation» (pp. 209-297), se concentre sur le travail de réécriture de l'oralité (sous forme d'emprunts thématiques aussi bien que de pratiques et de structures typiques des récits oraux) dans l'œuvre de Kourouma. Mestaoui aborde la question de la voix narrative dans les romans kouroumiens en analysant les procédés stylistiques - tels que l'emploi des incidentes (elle en recense cent vingt-deux dans les seuls Soleils), des apostrophes du narrateur à son auditoire et du discours indirect libre - par lesquels le romancier tâche de recréer la scène d'énonciation traditionnelle et l'expressivité de la parole orale. Mestaoui revient aussi sur l'éclatement des voix narratives qui a lieu dans un roman comme Monné, où «le narrateur anonyme à la Balzac partage sa narration avec une multitude de narrateurs occasionnels» (p. 223). L'auteur se concentre ensuite sur trois aspects de 
l'œuvre: la réactualisation des propriétés énonciatives du genre épique du donsomana qui a lieu dans En attendant le vote des bêtes sauvages; le paradigme de composition formulaire qui structure, de façon de plus en plus explicite du premier jusqu'au troisième roman, le récit kouroumien; l'emploi du proverbe et sa dimension métaphorique.

5 La dernière section de cette partie est réservée à la thématique du voyage. Mestaoui montre en quoi les trajectoires romanesques de Maclédio et de Koyaga (En attendant le vote des bêtes sauvages) peuvent être inscrites dans le modèle du voyage initiatique (qui suppose que le héros quitte un lieu social pour rejoindre un espace asocial d'où il pourra revenir seulement s'il surmonte une épreuve d'initiation) et du voyage épique (articulé autour de deux axes majeurs, l'errance et la conquête - ou la reconquête). En conclusion, on peut bien dire que Mestaoui atteint son but de montrer l'importance et l'étendue de l'influence de la tradition orale dans l'œuvre d'Ahmadou Kourouma, tout en soulignant, par sa stratégie de lecture, comment la réélaboration des modèles archétypaux a pu servir une représentation radicalement critique de la réalité contemporaine. 